\title{
Observation of a Change of Vibrational Excitation Mechanism with Surface Temperature: HCl Collisions with $\mathrm{Au}(111)$
}

\author{
Q. Ran, ${ }^{1}$ D. Matsiev, ${ }^{1}$ D. J. Auerbach, ${ }^{2}$ and A. M. Wodtke ${ }^{1, *}$ \\ ${ }^{1}$ Department of Chemistry and Biochemistry, University of California, Santa Barbara, California 93106-9510, USA ${ }^{\dagger}$ \\ ${ }^{2}$ Hitachi Global Storage Technologies, 4303 Yerba Buena Road, San Jose, California 95135, USA
}

(Received 1 December 2006; published 8 June 2007)

\begin{abstract}
We have measured the vibrational excitation probability $\left(P_{v}\right)$ of $\mathrm{HCl}$ incident on a $\mathrm{Au}(111)$ surface at kinetic energies $\left(E_{i}\right)$ of $0.59 \mathrm{eV}$ to $1.37 \mathrm{eV}$ and surface temperatures $\left(T_{s}\right)$ of $273 \mathrm{~K}$ to $1073 \mathrm{~K}$. For all energies, the slope of the $P_{v}$ as a function of $T_{s}$ exhibits a sharp increase above $T_{s} \approx 800 \mathrm{~K}$. We show this change in slope and the threshold behavior of $P_{v}$ to be consistent with a change in excitation mechanism from an electronically adiabatic mechanical mechanism to an electronically nonadiabatic mechanism involving excited electron-hole pairs.
\end{abstract}

The study of energy transfer dynamics at gas-solid interfaces strives to reveal an atomistic picture of energy flow in molecular collisions with surfaces, which is essential to understanding processes such as sticking, diffusion, and reactivity. Both experiment and theory have probed many aspects of such interactions including translational, vibrational, and rotational energy transfer between gasphase molecules and surface atoms [1-6]. Since the flow of energy into and out of chemical bonds is essential to bond rupture and formation, vibrational energy transfer takes a prominent place in this field [7-12]. For example, vibrationally inelastic scattering can be a sensitive probe of portions of the potential surface near the dissociation barrier [13]. Theoretical studies show that comparison of data on vibrational energy transfer and reactivity can even yield sensitive information on whether these two processes take place at different sites on the surface [13,14].

One of the most remarkable discoveries concerning vibrational energy transfer is the realization that molecular vibration may interact directly with electron-hole pair (EHP) excitations of the solid, destroying (creating) EHPs through vibrational excitation (relaxation) [15-23]. Such "electronically nonadiabatic" vibrational energy transfer is qualitatively different than "mechanical" (electronically adiabatic) energy transfer, which is dictated by the interatomic forces represented by a single potential energy hypersurface. In the adiabatic case, the energy reservoir for vibrational excitation must be the incidence energy of translation, $E_{i}$. This gives rise to a threshold in the dependence of vibrational excitation on $E_{i}$. In addition, the near harmonic nature of phonon motion and the low probability to couple multiple phonons to molecular vibration leads to a weak or absent dependence on $T_{s}$ [12]. Electronically nonadiabatic mechanisms for vibrational excitation, in contrast, may exhibit little or no incidence energy threshold as well as a characteristic pseudo-Arrhenius $T_{s}$ dependence resulting from the strong $T_{s}$-dependent variation of the Fermi function $[11,17]$. More recent evidence for the efficient coupling of intramolecular vibrational motion to
EHP excitations comes from the study of interactions of highly vibrationally excited molecules with metal surfaces. The observation of efficient multiquantum vibrational relaxation is hard to explain without invoking EHP excitation [24]. Taken together, these experiments provide strong indirect evidence for the role of EHP excitations. It has even been possible to provide direct evidence by the observation of efficient emission of electrons when $\mathrm{NO}(v=$ 18) molecules interact with a low work function surface [21].

Although existing evidence supporting the electronically nonadiabatic mechanism seems compelling, the role of thermally excited EHPs in vibrational excitation remains controversial. Holloway and co-workers [25,26] and Brenig and co-workers [27,28] have shown that it is possible to construct adiabatic models that reproduce the strong kinetic energy dependence and pseudo-Arrhenius $T_{s}$ dependence seen in the vibrational excitation of NO. Furthermore, vibrational excitation showing a strong $T_{s}$ dependence has so far only been seen for NO, making it difficult to assess the general importance of an EHP-V mechanism. Since both adiabatic and nonadiabatic mechanisms can contribute to vibrational excitation, it is desirable to find systems and circumstances where both mechanisms play a role and where a transition from an adiabatic to a nonadiabatic regime might be observed, thus providing a benchmark for theories of the relative importance of these two mechanisms.

In a previous paper, we reported the direct vibrational excitation of $\mathrm{HCl}$ in collisions with $\mathrm{Au}(111)$ at $273 \mathrm{~K}$ and presented evidence showing that a mechanical translational-to-vibrational energy transfer mechanism was at play [29]. Here, we extend the previous work to higher surface temperatures $T_{s}(273-1073 \mathrm{~K})$ and $E_{i}$ $(0.59-1.37 \mathrm{eV})$. As $T_{s}$ is increased, we observe a sharp increase in the slope of the vibrational excitation probability as a function of $T_{s}$ and a marked reduction in the previously reported translational energy threshold for vibrational excitation. We interpret these observations as a 
transition from an adiabatic to a nonadiabatic vibrational excitation mechanism with increasing $T_{s}$ and are able to quantitatively separate the two contributions to the energy transfer. These results provide an important benchmark as successful theories must treat adiabatic and nonadiabatic interactions on an equal footing.

The experiment has been described previously [29]. Jetcooled molecular beams are generated by expanding $\mathrm{HCl}$ seeded in $\mathrm{H}_{2}$ through a homemade piezoelectrically actuated pulsed valve. The expanded skimmed $\mathrm{HCl}$ beam passes through two differential pumping stages into the UHV surface chamber to collide with a gold surface. The short distance from nozzle to surface $(180 \mathrm{~mm})$ provides an intense molecular beam and the ability to time resolve incoming and scattered signals. The surface was cleaned daily by $\mathrm{Ar}^{+}$sputtering with subsequent anneals at $780 \mathrm{~K}$. Auger electron spectroscopy was used to monitor the surface condition. $\mathrm{HCl}(v=1)$ molecules were probed state selectively by $2+1$ resonance enhanced multiphoton ionization (REMPI) via the $Q$ branch of the $E^{1} \Sigma^{+}-$ $X^{1} \Sigma^{+}(0,1)$ band using $4-6 \mathrm{~mJ} /$ pulse tunable light near $247 \mathrm{~nm}$. The laser beam was focused with a $30 \mathrm{~cm}$ focal length lens. Laser generated ions were collected and guided by a repeller and two cylindrical focusing lenses to a microchannel plate detector. The incident and scattered molecules were detected $20 \mathrm{~mm}$ from the surface with different delay times (20-30 $\mu \mathrm{s})$ between the probe laser and the pulsed molecular beam. The short duration of our pulsed molecular beam $(<50 \mu \mathrm{s})$ provides some discrimination against "in beam background" even for these small delays. Rotational distributions were recorded by scanning laser wavelength, angular distributions were measured by translating the laser position along the line perpendicular to the molecular beam direction, and temporal profiles were obtained by recording the signal as a function of the delay between the laser with the nozzle.

Such data were obtained under 26 different conditions: six surface temperatures, $T_{s}=273,473,673,873,973$, and $1073 \mathrm{~K}$, for four values of $E_{i}=1.37,1.12,0.86$, and $0.59 \mathrm{eV}$ as well as a room temperature surface at $E_{i}=1.37$ and $1.12 \mathrm{eV}$. The scattered molecules' angular distributions all peak at the specular angle of $6.5^{\circ}$, due to the slightly $\left(3.3 \pm 0.3^{\circ}\right)$ off-normal incidence of the incoming molecular beam. Angular distributions of scattered $\operatorname{HCl}(v=1)$ are all quasispecular and broaden gradually with increasing $T_{s}$ due to surface atom thermal motion [30]. These observations are characteristic of direct "one bounce" interactions of $\mathrm{HCl}$ with the $\mathrm{Au}(111)$ surface.

To obtain absolute excitation probabilities [29], we compare the observed $v=1$ signal resulting from scattering to the thermal population in the incident molecular beam. The observed relative signals were corrected for differences in angular, rotational, and temporal distribution, as well as for laser power. The derived $T_{s}$ dependence of the vibrational excitation probability of $\mathrm{HCl}(v=0 \rightarrow$ 1 ) in collisions with $\mathrm{Au}(111)$ is shown in Fig. 1. Previously published results for $\mathrm{NO}(v=0 \rightarrow 1)$ on $\operatorname{Ag}(111)$ [17] are also shown. The solid or dashed straight lines are Arrhenius predicted calculations with effective activation energies equal to the vibrational excitation energy. There are several noteworthy observations. First, the vibrational excitation probabilities of $\mathrm{HCl}(v=0 \rightarrow 1)$ on $\mathrm{Au}(111)$ over the $T_{s}$ from 273 to $1073 \mathrm{~K}$ are in the range of $10^{-6}-10^{-4}$. In comparison to $\mathrm{NO}(v=0 \rightarrow 1)$ on $\operatorname{Ag}(111)$ where the largest excitation probability for NO is as high as $\sim 0.07$ [11,17], it is clear that the energy transfer is much less efficient for $\mathrm{HCl} / \mathrm{Au}$ compared to $\mathrm{NO} / \mathrm{Ag}$. Second, the vibrational excitation probabilities of $\mathrm{HCl}(v=0 \rightarrow 1)$ on $\mathrm{Au}(111)$ exhibit two different regimes of $T_{s}$ dependence. At low $T_{s}(273 \mathrm{~K}$ to $873 \mathrm{~K})$ the vibrational excitation is nearly independent of $T_{s}$. At higher values of $T_{s}$, the vibrational excitation probability $P_{v}$ is strongly temperature dependent and follows an Arrhenius law

$$
P_{v}=p\left(E_{i}\right) e^{E_{a} / k T_{s}}
$$

with an effective activation energy $E_{a}$ equal to the $\mathrm{HCl}(v=0-1)$ vibrational spacing, $E_{\mathrm{vib}}=0.36 \mathrm{eV}$. It has been previously shown that the population of EHPs with energy exceeding $E_{\text {vib }}$ is given by Eq. (1), assuming a metal whose density of states follows the Fermi function [17]. Thus, a pseudo-Arrhenius relation for vibrational excitation arises naturally for a mechanism involving the production of vibrational excitation via the deexcitation of thermal electron-hole pairs in the metal. We interpret the change in the degree of $T_{s}$ dependence from a nearly $T_{s}$-independent region to an Arrhenius dependence as a sign of a transition between two fundamentally different energy transfer mechanisms. In a previous paper, the ob-

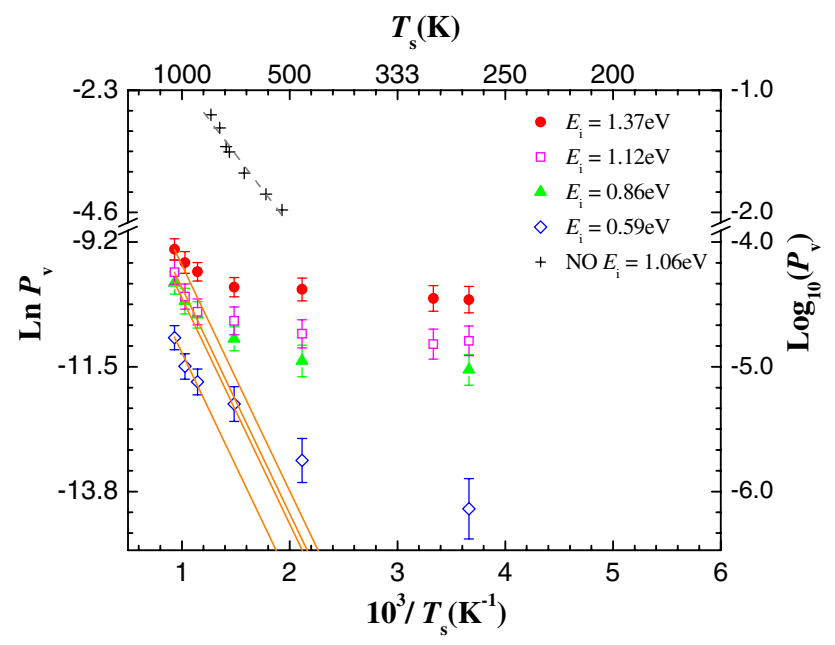

FIG. 1 (color online). $T_{s}$ dependence of vibrational excitation probability of $\mathrm{HCl}(v=0 \rightarrow 1)$ from $\mathrm{Au}(111)$ for 4 incidence energies. Comparison to NO data is also shown (from Ref. [17] ). The Arrhenius-like dependencies with the expected $E_{a}$ (slope) fixed at the $\mathrm{HCl} v=0-1$ energy spacing $(0.36 \mathrm{eV})$ are shown as solid lines. The Arrhenius dependence expected for NO with $E_{a}$ (slope) set by the vibrational spacing in $\mathrm{NO}(0.23 \mathrm{eV})$ is shown as a dashed line. 
served incidence energy threshold at $E_{i} \sim 0.57 \mathrm{eV}$ as well as a collapsing angular distribution near threshold were taken as strong evidence for a mechanical energy transfer mechanism at $T_{s}=273 \mathrm{~K}$ [29]. In light of previous work $[17,18]$, that the vibrational excitation probability follows the form of Eq. (1), we propose that this mechanism (EHPVenergy transfer) is operative for $\mathrm{HCl} / \mathrm{Au}$ scattering in the high $T_{s}$ limit of our data set. This line of reasoning allows us to separate the adiabatic and nonadiabatic contributions to $P_{v}$ using a simple model,

$$
P_{v}=P_{v}^{(1)}\left(E_{i}\right)+P_{v}^{(2)}\left(E_{i}, T_{s}\right)=p_{1}\left(E_{i}\right)+p_{2}\left(E_{i}\right) e^{-E_{a} / k T_{s}} .
$$

Here, $p_{1}\left(E_{i}\right)$ is the adiabatic excitation probability assuming $T_{s}$ independence [12]. The second term is the nonadiabatic excitation, where $E_{a}$ was fixed at the $\mathrm{HCl}(v=0-1)$ energy spacing $(0.36 \mathrm{eV})$. The $p_{2}\left(E_{i}\right)$ factor reflects the inherent coupling of metal electrons to the scattering molecule's vibrational motion and depends only on $E_{i}$, following [17] and references therein. The results of using this model to fit the data from all 26 experiments are shown in Fig. 2. These fits allow us to extract values of $p_{1}\left(E_{i}\right)$ (adiabatic) and $p_{2}\left(E_{i}\right)$ (nonadiabatic) for all 26 experimental conditions. Note that we need make no assumption concerning the $E_{i}$ dependence of $p_{1}\left(E_{i}\right)$ or $p_{2}\left(E_{i}\right)$; however, this analysis leads to the conclusion that both $p_{1}\left(E_{i}\right)$ and $p_{2}\left(E_{i}\right)$ are approximately linearly dependent on $E_{i}$. More specifically, $p_{1}\left(E_{i}\right)$ is linear with a threshold near $E_{i}=0.57 \mathrm{eV}$, similar to Fig. 4 of Ref. [29], while $p_{2}\left(E_{i}\right)$ is linear with no energy threshold (i.e., $y$ intercept $=0$ ).

Using these parameters we may calculate the relative contributions of adiabatic and nonadiabatic energy transfer $P_{v}^{(1)}\left(E_{i}\right)$ and $P_{v}^{(2)}\left(E_{i}, T_{s}\right)$ for 24 experiments at 6 surface

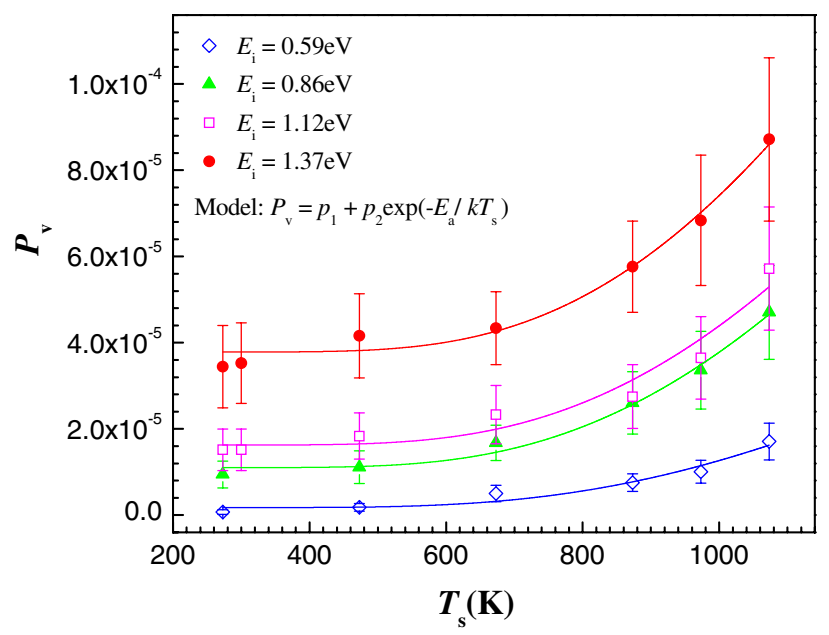

FIG. 2 (color online). Model simulation of $v=0 \rightarrow 1$ vibrational excitation of $\mathrm{HCl}$ on $\mathrm{Au}(111)$. The symbols are the experimental measurements and the solid curves are the simulated results. Combined adiabatic and nonadiabatic mechanisms are used in the model. temperatures $\left(T_{s}=298 \mathrm{~K}\right.$ results have been left out for clarity) as shown in Fig. 3. The influence of $E_{i}$ and $T_{s}$ variation on the total $P_{v}$ is shown in Fig. 3(a). Here the symbols are observation and the solid lines are the modeled values. The derived adiabatic and nonadiabatic contributions to the $P_{v}$ are shown in Fig. 3(b) and 3(c), respectively. Thus, for a specific choice of $T_{s}$ and $E_{i}$, the value of Fig. 3(a) is the sum of the values of Fig. 3(b) and 3(c). One can see that the adiabatic contribution, Fig. 3(b), depends monotonically on $E_{i}$ and is independent of $T_{s}$ within experimental error. There is a marked threshold $\left(E_{i} \sim 0.57 \pm 0.07 \mathrm{eV}\right)$ indicating conversion of $E_{i}$ to molecular vibration, rotation, and surface excitation. As has been pointed out previously, the threshold is substantially higher than $\mathrm{HCl}(v=0 \rightarrow 1)$ vibrational spacing $\left(E_{\mathrm{vib}}=\right.$ $0.36 \mathrm{eV})$, reflecting the inevitable excitation of surface degrees of freedom when a high energy projectile encounters an Au surface [29]. Of particular note, the nonadiabatic component exhibits no incidence energy threshold and strong $T_{s}$ dependence, whereas the adiabatic component exhibits a translational energy threshold and weak or absent $T_{s}$ dependence. At low values of $T_{s}$, the nonadiabatic contribution is nearly absent; the excitation is dominated by an electronically adiabatic mechanism. With increasing $T_{s}$, the nonadiabatic excitation increases rapidly. It is in-

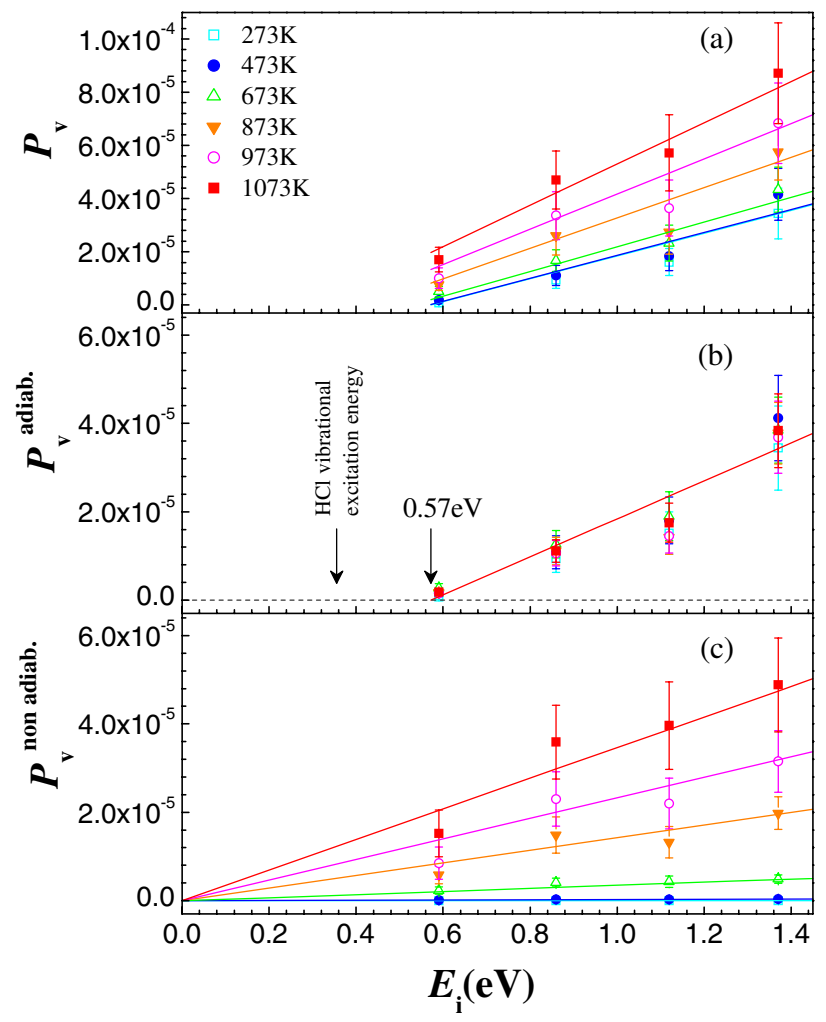

FIG. 3 (color online). $\quad E_{i}$ dependence of vibrational excitation probability of $\mathrm{HCl}(v=0 \rightarrow 1)$ from $\mathrm{Au}(111)$ at 6 values of $T_{s}$. (a) Total vibrational excitation. (b) Adiabatic vibrational excitation. (c) Nonadiabatic vibrational excitation. The solid lines are the model calculations. $T_{s}=298 \mathrm{~K}$ data have been left out of the figure for clarity. 
teresting to speculate that a high temperature regime may exist for many systems where molecule-surface interactions are dominated by electronically nonadiabatic influences. It is important to realize that in an EHP-V mechanism, the activation energy is expected to be independent of incidence energy and equal to the vibrational energy spacing $(0.36 \mathrm{eV})$. While it is possible to obtain a pseudoArrhenius $T_{s}$ dependence for a model phonon-to-vibration (adiabatic) energy transfer mechanism, the pseudoArrhenius activation energy in these models decreases $[25,26]$ (in one model linearly [31]) with increasing $E_{i}$. Furthermore, activation energies predicted from theory for $\mathrm{NO}$ on $\mathrm{Ag}$ and $\mathrm{H}_{2}$ on $\mathrm{Cu}$ for a phonon-mediated vibrational excitation mechanism are typically less than $0.1 \mathrm{eV}$ and decrease with increasing $E_{i}$. In addition, phonon-mediated models have not been reported to exhibit as large a curvature in the Arrhenius plot as our data over the temperature range shown in Fig. 1 or a thresholdless incidence energy dependence as is seen in Fig. 3. Thus, it appears unlikely that the phonon-mediated mechanism could explain all of the observations of this work.

The ability to separate the nonadiabatic and adiabatic contributions to $P_{v}$ allows us to quantitatively compare the nonadiabatic contribution for different molecule-surface systems. For example, comparison of $A_{\mathrm{HCl} / \mathrm{Au}}\left(E_{i}=\right.$ $1.12 \mathrm{eV}) \sim 1.9 \times 10^{-3}$ with $A_{\mathrm{NO} / \mathrm{Ag}}\left(E_{i}=1.06 \mathrm{eV}\right) \sim 1.8$ shows that electronically nonadiabatic effects are about 1000 times smaller for $\mathrm{HCl} / \mathrm{Au}$ than $\mathrm{NO} / \mathrm{Ag}$. There is no apparent reason why systematic evaluations of nonadiabatic propensity cannot be generally measured in this way. While we have no predictive theory to explain these results, Newns [32] and others have postulated that the coupling between metal electrons and molecular vibration is strongly related to the energy of the affinity level near the point of closet approach. Relative to NO, the affinity level of $\mathrm{HCl}$ is energetically higher (electron affinity: $0.026 \mathrm{eV}$ for $\mathrm{NO}$ and $-0.5 \mathrm{eV}$ for $\mathrm{HCl}$ ). Thus, it can perhaps be qualitatively understood why $\mathrm{HCl}$ vibration would be less strongly coupled to the metal electrons compared to NO.

In conclusion, we report direct vibrational excitation of $\mathrm{HCl}(v=0 \rightarrow 1)$ in the collisions with a $\mathrm{Au}(111)$ surface with a probability of the order of $10^{-4}-10^{-6}, 2-3$ orders of magnitude smaller than analogous vibrational excitation probability of $\mathrm{NO}$ on noble metals. We observe a transition from a regime with a nearly $T_{s}$ independent excitation probability at low $T_{s}$ to a regime with a strongly $T_{s}$ dependent excitation probability at high $T_{s}$. The results are quantitatively consistent with a simple model involving a transition from an electronically adiabatic mechanism at low $T_{s}$ to the onset of an electronically nonadiabatic mechanism at high $T_{s}$. We believe this is the first observation of a transition from electronically adiabatic to nonadiabatic behavior in a molecule-surface collision system and hope the results will provide a benchmark for theories of molecular interactions with surfaces.
We gratefully acknowledge the financial support from DOE Office of Basic Energy Sciences Grant No. DEFG02-03ER1544 and Partnership for International Research and Education-for Electronic Chemistry and Catalysis at Interfaces - NSF Grant No. OISE-0530268.

*Corresponding author.

Email address: wodtke@chem.ucsb.edu

${ }^{\dagger}$ Electronic address: http://www.chem.ucsb.edu/ wodtke/ groupwebpage/

[1] J.A. Barker and D. J. Auerbach, Surf. Sci. Rep. 4, 1 (1984).

[2] R. B. Gerber, Chem. Rev. 87, 29 (1987).

[3] C. R. Arumainayagam and R. J. Madix, Prog. Surf. Sci. 38, 1 (1991).

[4] M. L. Yu and L. A. Delouise, Surf. Sci. Rep. 19, 285 (1994).

[5] C. T. Rettner et al., J. Phys. Chem. 100, 13021 (1996)

[6] A. M. Wodtke, J.C. Tully, and D. J. Auerbach, Int. Rev. Phys. Chem. 23, 513 (2004).

[7] A. M. Wodtke, H. Yuhui, and D. J. Auerbach, Chem. Phys. Lett. 413, 326 (2005).

[8] C. T. Rettner, D. J. Auerbach, and H. A. Michelsen, Phys. Rev. Lett. 68, 2547 (1992).

[9] Z. H. Liu et al., Science 312, 1024 (2006).

[10] H. Hou et al., Science 284, 1647 (1999).

[11] C. T. Rettner et al., Surf. Sci. 192, 107 (1987).

[12] B. D. Kay, T. D. Raymond, and M. E. Coltrin, Phys. Rev. Lett. 59, 2792 (1987).

[13] G. R. Darling and S. Holloway, J. Chem. Phys. 97, 734 (1992).

[14] G. J. Kroes et al., Phys. Rev. B 53, 10397 (1996).

[15] M. Morin, N. J. Levinos, and A. L. Harris, J. Chem. Phys. 96, 3950 (1992).

[16] J. T. Kindt et al., J. Chem. Phys. 109, 3629 (1998).

[17] C. T. Rettner et al., Phys. Rev. Lett. 55, 1904 (1985).

[18] E. K. Watts, J. L. W. Siders, and G. O. Sitz, Surf. Sci. 374, 191 (1997).

[19] Y. Huang et al., Phys. Rev. Lett. 84, 2985 (2000).

[20] Y.H. Huang et al., Science 290, 111 (2000).

[21] J. D. White et al., Nature (London) 433, 503 (2005).

[22] L. Diekhoner et al., J. Chem. Phys. 117, 5018 (2002).

[23] A. C. Luntz, M. Persson, and G. O. Sitz, J. Chem. Phys. 124, 091101 (2006).

[24] N. Shenvi et al., J. Chem. Phys. 125, 154703 (2006).

[25] G. A. Gates, G. R. Darling, and S. Holloway, J. Chem. Phys. 101, 6281 (1994).

[26] G. A. Gates and S. Holloway, Surf. Sci. 309, 132 (1994).

[27] A. Gross and W. Brenig, Surf. Sci. 302, 403 (1994).

[28] A. Gross, Y. Chiba, and W. Brenig, Prog. Theor. Phys. Suppl. 106, 335 (1991).

[29] Q. Ran et al., Nucl. Instrum. Methods Phys. Res., Sect. B 258, 1 (2007).

[30] F. O. Goodman and H. Y. Wachman, Dynamics of GasSurface Scattering (Academic, New York, 1976).

[31] Z.S. Wang, G. R. Darling, and S. Holloway, J. Chem. Phys. 120, 2923 (2004).

[32] D. M. Newns, Surf. Sci. 171, 600 (1986). 